\title{
REVIEW
}

\section{Update on childhood rhabdomyosarcoma}

\section{H P McDowell}

The overall survival of childhood rhabdomyosarcoma has improved dramatically over the past 10 years. Early diagnosis and appropriate referral to a specialised centre leading to an accurate and timely diagnosis reflects on overall outcome. Recent molecular studies have identified different biological subtypes resulting in the recognition of poorer subgroups and allowing more appropriate treatment to be administered. Clinical trials remain the cornerstone to further improve outcome, now carried out on an international basis.

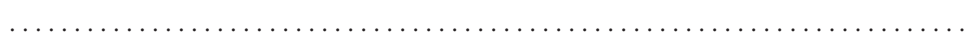

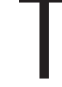

hat ubiquitous lump for which an opinion is sought, having appeared "from nowhere" or associated with a history of trauma, remains a diagnostic challenge. Although the majority of these lumps will prove innocent, some resolving spontaneously, the possibility of a soft tissue sarcoma should always have a place in the list of differential diagnoses. The most common soft tissue sarcoma of childhood is rhabdomyosarcoma (RMS). About 60 new cases of RMS are diagnosed in the UK each year. This accounts for approximately two thirds of all sarcomas in children aged 0-14 years, and $7-8 \%$ of malignant solid tumours in childhood. ${ }^{1}$ This frequency is the same as for Wilms' tumour and neuroblastoma. The incidence of RMS is highest in children $1-4$ years of age, falling to a lower rate at 10-14 years, and remains steady at 15-19 years of age. Histologically there are two major types of RMSembryonal (about 75\% of RMS cases) and alveolar-which tend to occur at different body sites and present in different age groups. The incidence of embryonal RMS is highest among younger children ( $0-4$ years), while alveolar RMS presents throughout childhood. ${ }^{2}$ RMS is a highly malignant tumour and is thought to arise from primitive mesenchymal cells committed to develop into striated muscle. ${ }^{3}$ It thus follows that this tumour can arise virtually anywhere in the body, including sites where healthy striated muscle would not normally be found.

Correspondence to: H P McDowell, RLC NHS Trust Alder Hey, Liverpool, UK

Dr H P McDowell, Consultant Paediatric Oncologist, RLC NHS Trust Alder Hey, Eaton Road, Liverpool L12 2AP, UK Heather.McDowell@ RLCH.NWEST.NHS.UK

Accepted 3 September 2002 is extension into contiguous organs, and in some cases, the presence of metastatic disease. Figure 1 shows the frequency of primary sites, and table 1 outlines the most common signs and presenting symptoms. In particular, RMS can present very indolently, with non-specific and minimal signs

\section{AETIOLOGY}

Epidemiological studies have indicated that for some RMS cases genetic factors may play an important part. An increase in both central nervous system anomalies and genitourinary abnormalities similar to those associated with Wilms' tumour is recognised. ${ }^{4}$ Other associations are with Gorlin's nevoid basal cell carcinoma, fetal alcohol syndrome, and neurofibromatosis. In 1969, Li and Fraumeni reported the increased incidence of breast carcinoma in mothers of children and young adults diagnosed with RMS. ${ }^{5}$ Further studies then identified the increased intrafamilial incidence of a distinct group of cancers: bone and soft tissue sarcomas, breast carcinoma, gliomas, leukaemia, and adrenocortical carcinomas, mostly occurring below the age of 45 years in affected families. Pedigree studies have revealed a dominant inheritance pattern, and those families are now known as having the well recognised Li-Fraumeni (or family cancer) syndrome. ${ }^{67}$ This dominantly inherited condition is associated with a faulty $p 53$ gene. $p 53$ is a tumour suppressor gene and plays a critical role in mediating cell cycle arrest or apoptosis (programmed cell death) in response to DNA damage: a transient arrest of the cell cycle in Gl phase with DNA repair, or apoptosis if DNA repair is not possible. ${ }^{8}$ When a family with a LiFraumeni pedigree is identified, genetic counselling should be offered to the parents and the patient, if they are deemed to be competent, to deal with the information.

\section{DIAGNOSIS AND STAGING}

When suspicion is aroused by a non-resolving lump, early referral to one of the recognised United Kingdom Children's Cancer Study Group (UKCCSG) centres is of paramount importance. Trocar or open biopsy in inexperienced hands cannot only lead to a failure of diagnosis, but also leave a contaminated site, whereby if second surgery is needed the least destructive approach may not include the original biopsy site. RMS is relatively rare and the histopathological diagnosis can be hard to make, particularly in recognising a small area of alveolar histology. Expertise can only be built up by regular exposure to these rare tumours, and histopathological investigations are best undertaken by pathologists familiar with paediatric tumours. Moreover, the need for frozen tissue is now of great importance in order to carry out molecular diagnostic investigations. and symptoms.

Abbreviations: EFS, event free survival; OS, overall survival; RMS, rhabdomyosarcoma 
Table 1 Common clinical symptoms of rhabdomyosarcoma

\begin{tabular}{ll}
\hline Head-neck & Asymptomatic mass, may mimick enlarged lymph node \\
Orbit & Proptosis, chemosis, ocular paralysis, eyelid mass \\
Nasopharynx & Snoring, nasal voice, epistaxis, rhinorrhoea, local pain, dysphagia, cranial nerve palsies \\
Paranasal sinuses & Swelling, pain, sinusitis, obstruction, epistaxis, cranial nerve palsies \\
Middle ear & Chronic otitis media, haemorrhagic discharge, cranial nerve palsies, extruding polypoid mass \\
Larynx & Hoarseness, irritating cough \\
Trunk & Asymptomatic mass (usually) \\
Biliary tract & Hepatomegaly, jaundice \\
Retroperitoneum & Painless mass, ascites, gastrointestinal or urinary tract obstruction, spinal cord symptoms \\
Bladder/prostate & Haematuria, urinary retention, abdominal mass, constipation \\
Female genital tract & Polypoid vaginal extrusion of mucosanguineous tissue, vulval nodule \\
Male genital tract & Painful or painless scrotal mass \\
Extremity & Painless mass, may be very small but with secondary lymph node involvement \\
Metastatic & Non-specific symptoms, associated with the diagnosis of leukaemia \\
\hline
\end{tabular}

Staging of a tumour requires a battery of investigations as shown in table 2. Specialised imaging is required, bone destruction being more visible on computed tomography scan and soft tissue masses on magnetic resonance imaging. Bone marrow biopsies are needed, and these can be performed at the same time that a central venous line is being placed for treatment, allowing coordination for both diagnosis and management of the patient.

\section{HISTOPATHOLOGY AND MOLECULAR GENETICS}

The two histopathological types of RMS commonly seen in paediatric practice are embryonal and alveolar. The latter can be characterised by specific genetic changes. ${ }^{9}$ The most consistent chromosome rearrangements to be identified in alveolar RMS are $\mathrm{t}(2 ; 13)(\mathrm{q} 35 ; \mathrm{q} 14)$ in $55 \%$ of cases and $\mathrm{t}(1 ; 13)(\mathrm{p} 36 ; \mathrm{q} 14)$ in about $22 \%$ of cases, ${ }^{10}$ as shown in fig 2 . The translocations involve two PAX genes, PAX3 and PAX7 located on chromosomes 2 and 1 respectively. These two genes are thought to be important in muscle development during

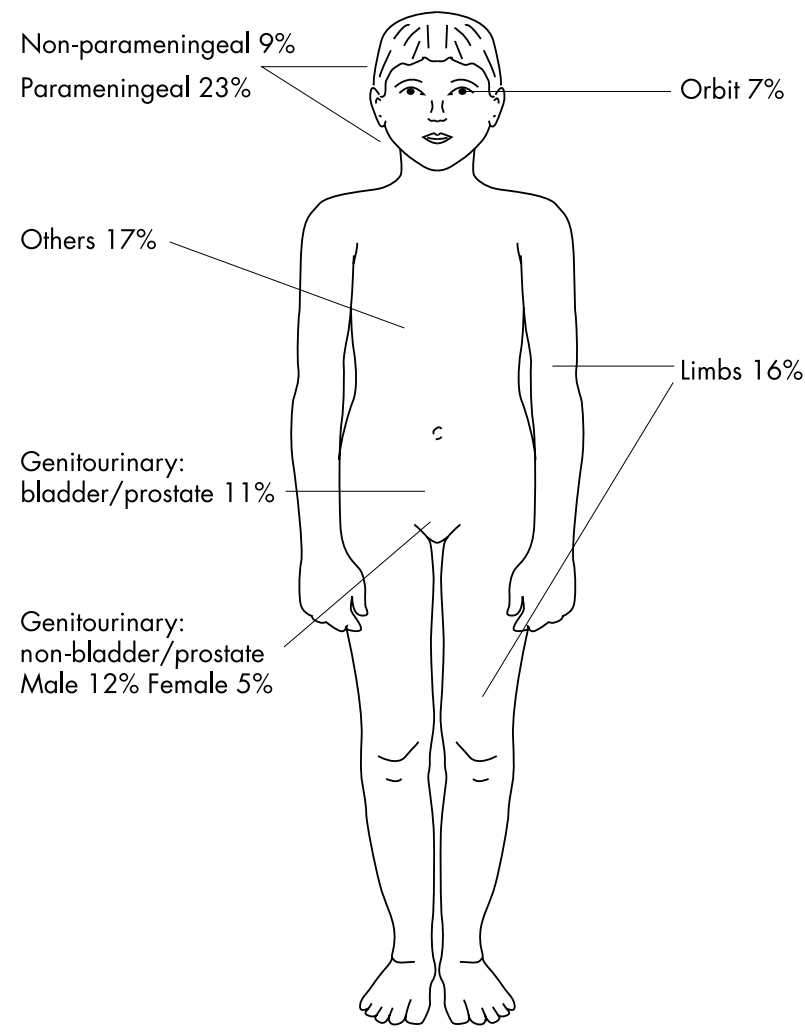

Figure 1 Distribution of RMS by primary site. embryogenesis, when condensations of mesoderm known as somites are formed from which skeletal muscle will develop. Disruption in the function of these genes can result in abnormal muscle development. Both translocations $\mathrm{t}(2 ; 13)(\mathrm{q} 35 ; \mathrm{q} 14)$ and $\mathrm{t}(1 ; 13)(\mathrm{p} 36 ; \mathrm{q} 14)$ result in fusion genes between the undisrupted PAX3 and PAX7 DNA binding domains, and the transactivation domain of the FKHR gene on chromosome 13. The resultant fusion genes, PAX3-FKHR and PAX7-FKHR, are expressed as chimeric transcripts that encode chimeric proteins and activate transcription from PAX binding sites with a higher potency than the corresponding wild type. ${ }^{11}$ These translocations result in an alteration of biological activity at protein level and are thought to influence tumorigenic behaviour by impacting on the control of tumour cell growth, apoptosis, differentiation, and motility. ${ }^{11}$ Both fusion genes provide a unique diagnostic marker for alveolar RMS, and can be used as an adjunct to classical histological diagnosis, ${ }^{10}$ particularly when this is proving difficult. This distinction is critical in that treatment options for alveolar and embryonal tumours are very different. A key question is whether the presence of a translocation leads to a worse prognosis. Analyses so far would suggest that PAX3-FKHR is associated with a poorer prognosis compared to PAX7-FKHR. ${ }^{10}{ }^{12}$ With regard to embryonal RMS, no specific molecular markers have been identified to date.

Other genetic changes presently under investigation in RMS include amplification of MYCN, MDM2, and CDK4, and chromosomal gains on 2, 8, 12, and 13. Loss of alleles and imprinting on 11 pl5.5 with disruption of several genes have also been implicated in RMS development. ${ }^{13}{ }^{14}$ Further investigation of these areas of interest will hopefully more accurately

Table 2 Diagnostic investigations

Blood

Full blood count, ESR, coagulation screen, group and save Renal and liver profile

Virology-cytomegalovirus, herpes zoster, measles titres Bone marrow aspirates and trephine

Constitutional DNA (for long term storage)

Cerebrospinal fluid (specific primary sites)

Glomerular filtration rate

Imaging

Chest $x$ ray

Ultrasound abdomen

CT scan - primary site/chest/abdomen/pelvis

$\mathrm{CT} / \mathrm{MR}$ brain for alveolar histology and limb primaries

MR for some specific primary sites

Bone scan

Echocardiogram (patients who will receive an anthracycline)

Audiometry (patients receiving carboplatin)

Biopsy (to include cytogenetics and long term storage)

$E S R$, erythrocyte sedimentation rate; $C T$, computed tomography; MR, magnetic resonance. 


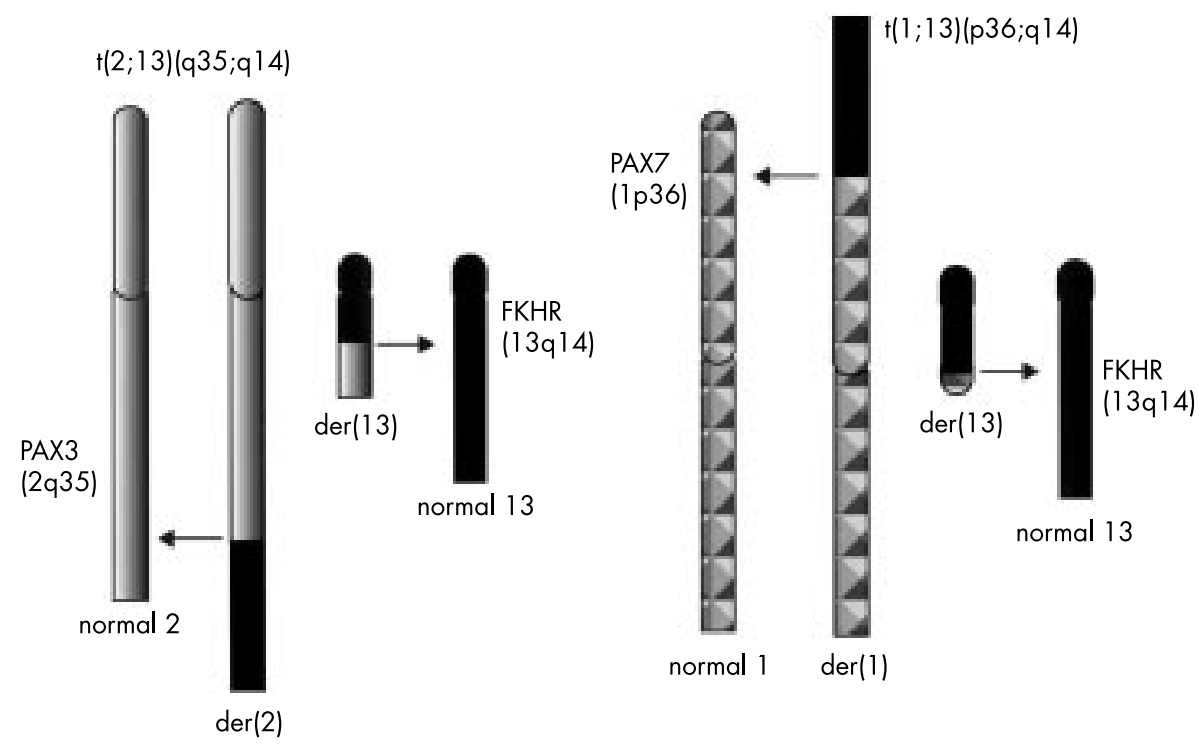

Figure 2 Diagram of the balanced chromosomal translocations resulting in the fusion genes involving PAX3 and PAX7 and the forkhead gene on chromosome 13.

delineate tumour behaviour on a biological basis and lead to more appropriate therapeutic strategies.

\section{TREATMENT}

The improved treatment for RMS over the last 20 years has resulted in an increase of overall survival (OS) from 25\% in 1970 to $75 \%$ in $1990 .{ }^{9}$ This has taken place within the forum of randomised clinical studies. The American Intergroup Rhabdomyosarcoma (IRS) Group opened their first study in 1972, while in Europe the International Society of Paediatric Oncology (SIOP) commenced their first trial in 1984. The Soft Tissue Sarcoma Group of the UKCCSG joined the SIOP group in 1989, and since that time there has been increasing international collaboration, now inclusive of Australasia and Argentina in the present study MMT95 for non metastatic disease. Entry of patients with RMS into clinical trials in the UK has risen from $21 \%$ in 1985 to $75 \%$ in 1995, with a resultant improvement of survival showing a significant trend of $\mathrm{p}<0.001$ as shown in fig 3 .

Over this time period, SIOP and IRS adopted two differing treatment philosophies to local control. The IRS has tended to use aggressive surgery and routine radiotherapy, except for the tumours totally excised at diagnosis. This is followed by prolonged chemotherapy regimens of up to two years. ${ }^{15}{ }^{16}$ The SIOP group has used chemotherapy to attain as many complete remissions as possible before using surgery and

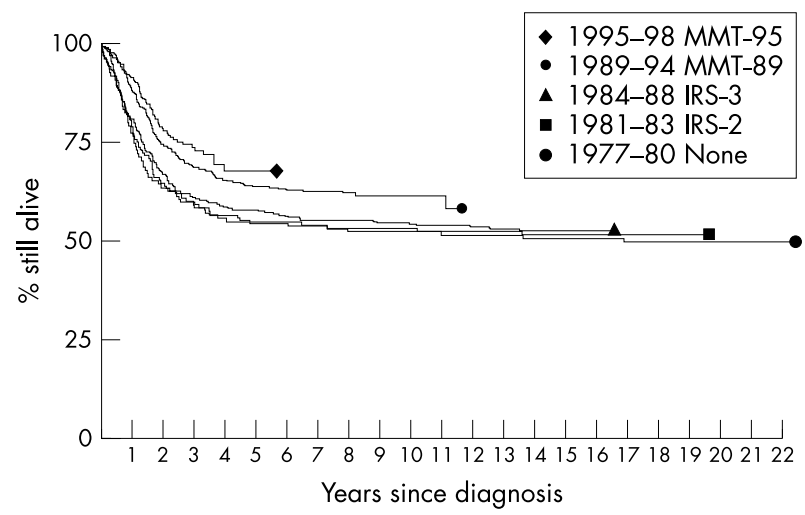

Figure 3 Survival of UKCCSG patients diagnosed 1977-98 by trial entry period. All stages included. radiotherapy for local control, in an attempt to avoid devastating late sequelae resulting from treatment. The overall chemotherapy treatment is much shorter, ${ }^{17-19}$ and in some cases is as little as nine weeks. The Italian and German working groups have adopted philosophies in between those of SIOP and IRS. All groups use the same cytotoxic agents, of which vincristine, actinomycin D, cyclophosphamide or ifosfamide, and etoposide form the backbone of the regimens given. ${ }^{17}$ Maturation of studies and dialogue between these groups is showing that most probably the ideal treatment lies somewhere between the different approaches. At present a dialogue is ongoing with our European colleagues in order to secure an European-wide study which will have a faster recruitment rate and help advance treatment more rapidly.

On completion of the diagnostic investigations, present treatment is based on a complex appraisal. The treatment protocols for RMS in the SIOP group are based on stratifying tumours according to: (1) primary tumour site: favourable sites include orbit, paratesticular, and vagina, while higher risk sites are parameningeal and limbs; (2) stage: totally excised tumours have a superior outcome, while residual disease post biopsy, nodal, or metastatic involvement result in a worse outcome; (3) histology: alveolar has a poorer outcome than embryonal. Recent analysis has also revealed that primary tumour size $<5 \mathrm{~cm}$ or $\geqslant 5 \mathrm{~cm}$ and age $<10$ years or $\geqslant 10$ years, are prognostic factors: larger tumours and an older age have a worse outcome (SIOP Group, unpublished data). In metastatic disease, bone and/or bone marrow involvement, hold a worse outcome along with age $>10$ years. These last three parameters have been taken into account in treatment stratification for patients treated in the present SIOP protocol for metastatic disease, MMT98. This study is investigating the use of sequential high dose monotherapy in high risk metastatic disease. Unfortunately, to date there is no robust evidence to support the use of high dose chemotherapy with stem cell rescue in the treatment of these patients to ensure an improved outcome..$^{20}$ The three year OS for patients with nonmetastatic disease is $86 \%{ }^{21}$ For low risk patients whose primary tumour is totally excised and arises from a favourable site, the expected three year OS and event free survival (EFS) is $94 \%$ and $89 \%$ respectively. High risk patients with unresectable tumours in unfavourable sites, for example, parameningeal tumours, have a three year OS and EFS of $73 \%$ and $67 \%$ respectively. ${ }^{22}$ In addition, many limb primaries remain unresectable and have early dissemination as there is a 
Practice points

- Regard non-resolving lumps as suspicious

- Early referral to an UKCCSG centre is needed prior to any biopsy

- Appropriate tissue collection to include frozen tissue for cytogenetic markers

- Overall survival at five years is $72 \%$

predominance of alveolar histology at this primary site. For these patients, three year OS and EFS are $66 \%$ and $62 \%$ respectively. ${ }^{21}$

\section{LATE EFFECTS}

The balance between cure and irreversible late sequelae as a result of treatment remains one of the biggest challenges in treating children with RMS. The acute side effects of cytotoxic chemotherapy, such as marrow suppression and immune suppression can now be largely managed with up to date supportive care strategies. However, as the OS of patients increases, late sequelae are becoming more apparent and important. ${ }^{23}{ }^{24}$ Cardiac toxicity from anthracycline exposure can occur in spite of close monitoring at the time of administration, but can appear some years later as congestive cardiac failure and may require a heart transplant. Platinum agents, for example, carboplatin can give rise to long term renal tubular acidosis, and result in high tone deafness with the need for hearing aids. Ifosfamide is associated with renal tubular acidosis, chronic urinary electrolyte loss, and renal rickets with osteoporosis. As an alkylating agent ifosfamide also has the potential for second primary malignancy development, potentiated if radiotherapy is also administered. The level of fertility, especially in males, is as yet undetermined after receiving this agent. Radiotherapy singularly has the potential for further primary tumour development, and when administered at a young age results in a substantial soft tissue and vascular hypoplasia. This may make a limb dysfunctional, can alter body image substantially, and have a devastating psychological effect, with the practical difficulties of finding suitably fitting clothes. Radiotherapy directed to the CNS at the dose required in children $<3$ years old can result in major learning difficulties and failure to achieve independence as an adult. Late effects as a result of surgery depend very much on the site and extent of the surgical procedure. For those good risk groups, the issue of quality of life after treatment has now become of prime importance.

\section{SUMMARY}

The OS for children diagnosed with RMS has improved considerably over the past decade. This reflects the intensification of chemotherapy within the setting of multimodal treatment. The contribution of randomised clinical trials undertaken in specialised centres in achieving this cannot be underestimated.$^{25}$ However, present public opinion is resulting in a significant number of parental refusals for their child to enter into these trials (unpublished data) and is threatening this form of investigative treatment. Trial analysis has allowed specific clinical treatment groups to be identified within this heterogeneous disease. Molecular analysis looks promising in unravelling the biological behaviour, leading to further refinement in treatment.

Timely referral to an appropriate treatment centre still remains the cornerstone for a favourable outcome and relies on the awareness of the referring physician.

\section{ACKNOWLEDGEMENTS}

We acknowledge Dr C Stiller and Dr N Birkin for reviewing the manuscript, and S Kennedy for help with the manuscript.

\section{REFERENCES}

$1 \mathrm{NCl}$. Cancer incidence and survival among children and adolescents: United States SEER Program 1975-1995. National Cancer Institute.

2 IARC. International incidence of childhood cancer, 1998 (IICC-2), Vol. II IARC Scientific Publication No. 144

3 Gaiger AM, Soule EH, Newton WA Jr. Pathology of rhabdomyosarcoma: experience of the Intergroup Rhabdomyosarcoma Study, 1972-1978. Natl Cancer Inst Monogr 1981;56:19-27.

4 Ruymann FB, Maddux HR, Ragab A, et al. Congenital anomalies associated with rhabdomyosarcoma: an autopsy study of 115 cases: a report from the Intergroup Rhabdomyosarcoma Study Committee. Med Pediatr Oncol 1988;16:33-9.

5 Li FP, Fraumeni JR. Rhabdomyosarcoma in children: epidemiologic study and identification of a family cancer syndrome. J Natl Cancer Inst 1969;43:1365-73

6 Li FP, Fraumeni JR, Mulvihill JT, et al. A cancer family syndrome in twenty-four kindreds. Cancer Res 1988;48:5358-62.

7 Birch JM, Hartely AL, Blair V, et al. Cancer in the families of children with soft tissue sarcoma. Cancer 1990;66:2239-48.

8 Bargonetti J, Manfredi JJ. Multiple roles of tumor suppressor p53. Curr Opin Oncol 2002;14:86-91.

9 Pappo AS, Shapiro DN, Crist WM, et al. Biology and therapy of pediatric rhabdomyosarcoma. J Clin Oncol 1995;13:2123-39.

10 Sorensen PHB, Lynch JC, Qualman SJ, et al. PAX3-FKHR and PAX7-FKHR gene fusions are prognostic indicators in alveolar rhabdomyosarcoma: a report from the Children's Oncology Group. $J$ Clin Oncol 2002;20:2672-9

11 Barr FG. Gene fusions involving PAX and FOX family members in alveolar rhabdomyosarcoma. Oncogene 2001;20:5736-46.

12 Kelly KM, Womer RB, Sorensen PH, et al. Common and variant gene fusions predict distinct clinical phenotypes in rhabdomyosarcoma. J Clin Oncol 1997;15:1831-6.

13 Anderson J, Gordon A, Pritchard-Jones K, et al. Genes, chromosome, and rhabdomyosarcoma. Genes Chrom Cancer 1999;26:275-85.

14 Gordon T, McManus A, Anderson J, et al. Cytogenetic abnormaltities in 42 rhabdomyosarcoma: a United Kingdom Cancer Cytogenetics Group Study. Med Pediatr Oncol 2001;36:259-67.

15 Breneman JC, Weiner ES. Issues in the local control of rhabdomyosarcoma. Med Pediatr Oncol 2000;35:104-9.

16 Baker KS, Anderson JR, Link MP, et al. Benefit of intensified therapy for patients with local or regional embryonal rhabdomyosarcoma: results from the Intergroup Rhabdomyosarcoma Study IV. J Clin Oncol 2000;18:2427-34

17 Martelli H, Oberlin O, Rey A, et al. Conservative treatment for girls with nonmetastatic rhabdomyosarcoma of the genital tract: a report from the study committee of the International Society of Pediatric Oncology. J Clin Oncol 1999;17:2117-22.

18 Baldini EH, Goldberg J, Jenner C, et al. Long-term outcomes after function-sparing surgery without radiotherapy for soft tissue sarcoma of the extremities and trunk. J Clin Oncol 1999;17:3252-9.

19 Oberlin O, Rey A, Anderson J, et al. Treatment of orbital rhabdomyosarcoma: survival and late effects of treatment-results of an international workshop. J Clin Oncol 2001;19:197-204

20 Carli M, Colombatti R, Oberlin O, et al. High-dose melphalan with autologous stem-cell rescue in metastatic rhabdomyosarcoma. J Clin Oncol 1999; 17:2796-803.

21 Crist WM, Anderson JR, Meza JL, et al. Intergroup Rhabdomyosarcoma Study-IV: results for patients with nonmetastatic disease. J Clin Oncol 2001;19:3091-102.

22 Raney RB, Meza J, Anderson JR, et al. Treatment of children and adolescents with localized parameningeal sarcoma: experience of the Intergroup Rhabdomyosarcoma Study Group protocols IRS-II through-IV, 1978-1997. Med Pediatr Oncol 2002;38:22-32.

23 Meister LA, Meadows AT. Late effects of childhood cancer therapy. Curr Probl Pediatr 1993;23:102-31.

24 Marina N. Long-term survivors of childhood cancer: the medical consequences of cure. Pediatr Clin North Am 1997:44:1021-42.

25 Stiller CA. Centralised treatment, entry to trials and survival. $\mathrm{Br} J$ Cancer 1994;70:352-62. 